\title{
Transforming Healthcare in Rural India by Telemedicine during COVID-19 Pandemic
}

\author{
Isha Srivastava ${ }^{1}$, Atil Kumar Lal ${ }^{2}$, Mahima Pandey ${ }^{3}$, Ashish Jaiswal ${ }^{4}$, Ishank Jaiswal ${ }^{5}$
}

\author{
${ }^{1}$ Department of Dentistry, All India Institute of Medical Sciences (AIIMS), Gorakhpur, Uttar Pradesh, India. \\ ${ }^{2}$ Department of Orthopaedics, All India Institute of Medical Sciences (AIIMS), Gorakhpur, Uttar Pradesh, India. ${ }^{3}$ \\ Department of Medicine, All India Institute of Medical Sciences (AIIMS), Gorakhpur, Uttar Pradesh, India. ${ }^{4}$ Department \\ of General Surgery, All India Institute of Medical Sciences (AIIMS), Gorakhpur, Uttar Pradesh, India. ${ }^{5}$ Department of \\ Otorhinolaryngology, All India Institute of Medical Sciences (AIIMS), Gorakhpur, Uttar Pradesh, India.
}

COVID-19 is a novel coronavirus, declared a global pandemic by World Health Organization (WHO) Emergency Committee. Coronavirus became a household name and the well-being of every health care worker is the cornerstone for proper functioning of health care systems in rural hospitals. The melancholy affecting the rural healthcare emerges from various vectors. Sparse population, equipment underuse and the absence of high margin specialty services, geographical locations desolate the economic outlook. When rural hospitals started using telemedicine, then there are more chances to get bypassed for the treatment at the urban facility, get remote consultation, in home monitoring, outsourced diagnostics analysis. It also reduces unnecessary footfalls to hospitals and clinics as it reduces cost by reducing readmissions and cut down on the time for specialty care and allow health care workers to enable "healing at distance". Rural hospitals with the help of telemedicine can change the landscape of rural physicians practice.

A global pandemic emerges and wreaks devastation on a susceptible world population once in a lifetime which leads to limited personal experience with such events. In the present scenario, there is an outbreak of coronavirus disease identified with intense respiratory illness called COVID-19 which was first announced in Wuhan, Hubei province in China in December 2019. On 11 th March 2020, World Health Organization (WHO) Emergency committee declared COVID-19 as a worldwide pandemic.

COVID-19 is rapidly expanding and has surpassed 26,121,999 confirmed cases and global death exceeds 864,618 worldwide. In India, total confirmed cases are $3,948,594$ and death exceeds to 68,682 . Since, it is a respiratory virus, patients who are suffering from asthma, chronic obstructive pulmonary disease, hypertension, diabetes and with immunodeficiency are the vulnerable groups at increased risk of morbidity. ${ }^{1}$ In this global pandemic, medical system is on the verge of a devastating collapse because of the over burden of the physicians and psychological distress including long work hours, sleep disturbances, debilitating fatigue which leads to caregiver burnout and shortage of medical practitioners. To overcome this situation, the world has woken up from its slumber and introduced the concept of "Forward Triage", the process of diagnosing the patient condition before their arrival in emergency department. ${ }^{2}$ To reduce unnecessary footfalls to hospitals and clinics and provide more manpower to healthcare systems, telemedicine became a critical asset as a valuable tool against this pandemic. A key factor to slow the transmission of virus is social distancing- "Healing at Distance" decreasing person to person contact. ${ }^{3}$

This article has outlined that telemedicine has acted as a natural evolution in healthcare in rural India during COVID-19 crisis.

\section{Telemedicine}

Even before the arrival of COVID-19, telemedicine existed for decades. It provides palliative care into the homes of the most vulnerable people and also saves valuable drive time for home visiting care. Earlier, the overall uptake of telemedicine was slow and fragmented because of heavy regulations and sparse supportive payment structures. ${ }^{4}$

\author{
Corresponding Author: \\ Dr. Atil Kumar Lal, \\ MS, All India Institute of Medical \\ Sciences, No. 282, Muktayan, \\ St. John's Church Road, Basharatpur, \\ Gorakhpur - 273004, Uttar Pradesh \\ India. \\ E-mail:atil27@gmail.com
}

DOI: $10.14260 / \mathrm{jemds} / 2020 / 813$

How to Cite This Article:

Srivastava I, Lal AK, Pandey M. Transforming healthcare in rural India by telemedicine during COVID-19 pandemic. J Evolution Med Dent Sci 2020;9(49):37033705, DOI: 10.14260/jemds/2020/813

Submission 20-08-2020,

Peer Review 23-10-2020,

Acceptance 28-10-2020,

Published 07-12-2020.

Copyright (c) 2020 Isha Srivastava et al. This is an open access article distributed under Creative Commons Attribution License [Attribution 4.0 International (CC BY 4.0)] 
Telemedicine is catapulted as an important device in this medical arsenal providing a first line defence and helps to mitigate the spread of COVID-19 despite it was not mainstreamed earlier. The potential of telemedicine is remote consultations, in home monitoring, remote specialists in rural areas and during this pandemic outbreak supporting triage, maintaining social distancing while reducing the risk of cross contamination caused by close contact. ${ }^{5}$

On 24th March 2000 telemedicine was started when Bill Clinton commissioned the world's first VSAT (ISRO supplied) enabled village hospital in Aragonda, Andhra Pradesh. Telemedicine has various brain-storming sessions, so proposals were put forwarded to NITI Ayog and other authorities to notify unbiased regulations. ${ }^{6}$

The Registered Medical Practitioner (RMP) follows the technology based tele-consultation under the Indian Medical Council (IMC) act 1956 and the guidelines framed by MOHFW. ${ }^{7}$

Telemedicine includes telephonic consultation, chat mode, messaging services, and SMS, using Electronic Medical Record (EMR) where case records, pictures, investigations, data can be stowed, recovered and a good video consultation can be done. ${ }^{6}$

\section{What is Tele-Triage?}

Tele-triage is the triage concept applied to the field of telemedicine to quickly assess the patient's condition remotely, for appropriate decision making. ${ }^{8}$

Acc. to MoHFW and Central and State Governments, PreTele-triage action includes a large variety of scenarios and therefore need to first ensure readiness of the remote advisor to accept the tele-triage request. It also includes equipment, Infrastructure, checklists, definitions and symptom complex criteria response scoring. Post tele-triage actions include technical, legal and clinical decisions and appropriate communication to patients and follow-up when necessary. ${ }^{8}$

Telemedicine can combat the COVID-19 outbreak and can bridge the gap between the rural-urban health systems. Rural hospitals with the help of telemedicine can change the landscape of rural physicians practice. According to FY 20162020 , telemedicine reaches upto $\$ 32$ million by 2020 (Indian e health market report 2020).

\section{Telemedicine as an Asset in Rural Areas in COVID - 19}

Reasons for success in rural areas could be scanty population, underutilization of equipment's, absence of high quality specialty services, higher percentage of uneducated patients, geographical locations and unfavourable economic outlook in rural areas. So, when rural hospitals will start using telemedicine, then there are more chances of remote consultation, in-home monitoring and outsourced diagnostics analysis in rural population. It will also lessen the unnecessary visits to hospitals / clinics and reduce re-admissions by lowering the patient's expenses.

\section{Advantages}

Remote consultations are faster, cheaper and more efficient than healthcare appointments. Indian Institute of Public Opinion reported that $89 \%$ of rural population has to travel at least $8 \mathrm{kms}$ to obtain the basic medical treatment. ${ }^{5}$ Poor Indian villagers spend most of their income in travelling to the urban hospitals and staying with their escorts for longer duration. Telemedicine can avoid these hassles of travel and reducing the waiting time outside the hospital. Consultations in remote areas are quicker, less expensive and more effective than hospital visits with the help of telemedicine.

Rural hospitals are utilizing telemedicine through in-home monitoring. Reducing the exposure of high risk individuals (elderly and with co-morbidities) to hospitals can flatten the curve of cross contamination in COVID-19. Before this pandemic outbreak, patients brush off common symptoms like flu and cold, but this sign of illness can be a new concern in this pandemic. ${ }^{5}$ Using CDC clinical guidelines, early diagnosis can be done with further escalation of care if required. ${ }^{9}$ As a result, early diagnosis will lead to substantial decline in unnecessary visits to hospitals for betterment of society, promoting selfquarantine.

Rural hospitals are also struggling in outsourced diagnostic analysis. Diagnosticians are not willing to stay in rural hospitals due to less resources and infrastructure, but mobile imaging centres can take x-rays and can work in conjunction with remote analysis labs in larger urban areas. ${ }^{9}$

Physicians can connect medical devices to the videoconference with the help of telemedicine solutions like V2MD to collect vital information for a rapid diagnosis. ${ }^{9}$ They can also evaluate shortness of breath, oxygen saturation and transmit heart and lung sounds through internet using digital stethoscope. 6

Telemedicine also helps in consultation with remote specialists, instead of having them as a staff. For example, if a patient is referred to pulmonologist, he / she fails to procure the treatment due to unavailability of that specialist in that area. People will avoid to seek treatment with specialist in urban areas and will stop taking medications for their follow ups. Through telemedicine, all specialists can discuss various options with the patient together leading to transparency at its highest. ${ }^{5}$

It also removes the barrier for the residents in geographical locations and isolated areas where public transport is sparse or in post disaster situations.

In COVID-19 situation, people in quarantine or selfisolation period have experienced anxiety, fear, loneliness, depression and have developed post-traumatic stress disorder (American Psychological Society, 2020: WHO). To conquer this, psychotherapy sessions can be conducted through telemedicine for the individuals. ${ }^{10}$ It can also be used for treatment monitoring even if the doctors and patients both are quarantined.

\section{Disadvantages}

In rural areas, telemedicine might take longer time in connecting virtual communications due to inadequate bandwidth to support transmission of data, images and sound. It leads to insufficient broadband infrastructure and poor wireless connection which cannot provide immediate treatment. Lack of funding is also responsible for slow growth of telemedicine. The overall cost including data management apparatus is also high as confidential medical information about patient can be leaked through faulty electronic systems. 
Medicare reimbursements is a major issue, individually having their own standards by which their Medicaid programs can reimburse for telemedicine expenses. Low quality of diagnostic informatics like X-rays, blood reports can be at the higher risk of faulty clinical treatment. Strict legal regulations should be made to avoid unauthorised and illegal services in remote sectors. ${ }^{5}$

\section{Challenges}

Rural hospitals have a pathway towards revitalization in the form of telemedicine, but hindrances still remain. In some areas, people revert to what they are used to doing and expects the way they previously interacted with the healthcare systems. Patients would desire their own doctor to be on telemedicine as they have a previously established relationship. It might be challenging for some healthcare workers also to learn new method of consulting and need special training in use of new technology.

To overcome these above mentioned barriers, we should educate the people that telemedicine is the safer and faster alternative under present circumstances, instruct the patients to operate step by step, reach individuals with poor social networks, less technological literacy, decrease the cost barriers to access telemedicine and expand network reimbursements coverage for the clinicians. ${ }^{1}$

\section{Conclusions}

Preparation, patience and practice is the hallmark for the success of the telemedicine. The success of any technology is based on several factors like skills, acquired attitude, knowledge and understanding of the concept. Telemedicine can be daunting to some individuals, but it is the need of the hour for better dissemination in this outbreak providing more manpower to the hospitals as a boosting engagement. WHO and other Global Healthcare Organizations should ensure equitable access to telemedicine technology for rural population through creative solutions and funding and provide a path for other countries to adopt and strengthen telemedicine services that will augment and optimize the planetary effort of COVID-19.

Financial or other competing interests: None.

Disclosure forms provided by the authors are available with the full text of this article at jemds.com.

\section{REFERENCES}

[1] Portnoy J, Waller M, Elliott T. Telemedicine in the era of COVID-19. J Allergy Clin Immunol Pract 2020;8(5):148991.

[2] Moazammi B, Khorasani NR, Moghadam AD, et al. COVID 19 and telemedicine: immediate action required for maintaining healthcare providers well-being. Journal of Clinical Virology 2020;126:104345.

[3] Strehle EM, Shabde N. One hundred years of telemedicine: does this new technology have a place in paediatrics? Arch of Dis Child 2006;91(12):956-9.

[4] Smith AC, Thomas E, Snoswell CL, et al. Telehealth for global emergencies: implications for coronavirus disease (COVID-19). J Telemed Telecare 2020:26(5):309-13.

[5] Ripton JT, Winkler CS. How telemedicine is transforming treatment in rural communities. Becker's Healthcare 2016;8:8.

[6] Ganapathy K. Coronavirus: will the crisis make virtual consultations go viral in India? 2020.

[7] Carlton B, Abedini N, Fratkin M. Telemedicine in the time of Coronavirus. J Pain Symptom Manage 2020;60(1):e124.

[8] Huibers L, Smits M, Renaud V, et al. Safety of telephone triage in out-of-hours care: a systematic review. Scand J PrimHealth Care 2011;29(4):198-209.

[9] Virk S. Combating the coronavirus with telemedicine. Health IT Outcomes Feb 25, 2020.

[10] Leite H, Hodgkinson IR, Gruber T. New development: 'healing at a distance'- telemedicine and COVID - 19. Public Money and Management 2020;40(6):483-5. 